\title{
Influência da posição de lâminas de duas espécies exóticas nas pro- priedades mecânicas de painéis LVL
}

\author{
Camila Berger $^{1 \star}$, Anderson Pertuzatti ${ }^{1}$, Clovis Roberto Haselein ${ }^{1}$ \\ ${ }^{1}$ Departamento de Ciências Florestais, Universidade Federal de Santa Maria (UFSM), Santa Maria, RS, Brasil.
}

\begin{abstract}
RESUMO O objetivo deste trabalho foi investigar a influência do posicionamento de lâminas de Hovenia dulcis e Corymbia citriodora em painéis estruturais LVL (Laminated Veneer Lumber) nas propriedades mecânicas: flexão estática nas posições flatwise e edgewise e cisalhamento na linha de cola ensaio a seco e após fervura. Para isso, foram avaliadas seis composições formadas por cinco lâminas de $1,0 \mathrm{~mm}$ de espessura coladas com adesivo fenol-formaldeído na gramatura de $200 \mathrm{~g} / \mathrm{m}^{2} \mathrm{em}$ linha simples, formando tratamentos puros e mistos. As composições foram submetidas à pré-prensagem a temperatura ambiente e, posteriormente, a prensagem a quente em prensa hidráulica na temperatura de $145^{\circ} \mathrm{C}$ e pressão específica de $14 \mathrm{kgf} / \mathrm{cm}^{2} . \mathrm{Os}$ resultados indicaram que houve maior influência da densidade dos painéis LVLs nos resultados de MOE e MOR na posição flatwise (exceto para o painel CHHHC- capa e contracapa de Corymbia citriodora e demais de Hovenia dulcis), do que as diferenças de densidade das lâminas que compõe os painéis, o inverso ocorre na posição edgewise. Na resistência ao cisalhamento a seco e após a fervura, a composição com apenas lâminas de Hovenia dulcis obteve o melhor desempenho, já a composição com mistura das espécies e com apenas lâminas de Corymbia citriodora apresentaram semelhante performance.

Palavras-chave: laminated veneer lumber; fenol formaldeído; Hovenia dulcis; Corymbia citriodora.
\end{abstract}

\section{Influence of the position of veneers of two exotic species on the mechanical properties of LVL panels}

\begin{abstract}
The aim of this work was investigate the influence of positioning wood veneers of Hovenia dulcis and Corymbia citriodora in structural panels LVL (Laminated Veneer Lumber) on the mechanical properties: static bending strength at flatwise and edgewise positions and shear strength in the glue line test dry and after boiling. Were evaluated six compositions formed by five wood veneers of $1,0 \mathrm{~mm}$ thick bonded with adhesive phenol formaldehyde in grammage of $200 \mathrm{~g} / \mathrm{m}^{2}$ in single line. They formed single and multiple species treatments. After subjected to pre-pressing at environmental temperature, they were then submitted to hot pressing in a hydraulic press at temperature of $145^{\circ} \mathrm{C}$ and specific pressure of $14 \mathrm{kgf} / \mathrm{cm}^{2}$. The results indicated that there is a greater influence of the density of LVL on the results of MOE and MOR in flatwise position (except for the CHHHC treatment - external layers of Corymbia citriodora and internal layers of Hovenia dulcis) than the differences in the density of woods veneers; in the edgewise position the opposite behavior occurred. In shear strength, the composition formed by just Hovenia dulcis veneers showed the best performance, and the others presented similar results.
\end{abstract}

Keywords: laminated veneer lumber; phenol formaldehyde; Hovenia dulcis; Corymbia citriodora..

\section{Introdução}

Apesar de culturalmente a madeira não ser um elemento importante na construção civil de casas, o Brasil por possuir uma alta produção de madeira tem potencial para competir com o mercado exterior na geração de produtos de madeira engenheirada, tais como os painéis estruturais, amplamente utilizados na América do Norte. Construções em madeira tem provado excelente performance a longo prazo, estão aliadas a sustentabilidade da cadeira produtiva por advirem de plantios reflorestáveis e, além disso, podem estocar $\mathrm{CO}_{2}$ da atmosfera. Painéis estruturais como o laminated veneer lumber (LVL) é o exemplo disso.

O LVL pertence ao grupo Structural Composite Lumber (SLC), caracterizado pela presença de lâminas coladas com a 
grã paralela umas às outras e com adesivo estrutural (fenolformaldeído) formando um compósito para fins estruturais. Em que as dimensões podem ser produzidas da mesma forma que usualmente é para os pranchões de madeira. Além disso, apresenta melhor desempenho mecânico que a madeira maciça, tanto no sentido perpendicular à face das lâminas (flatwise) quanto no sentido paralelo às mesmas (edgewise) e pode ser produzido para suportar grandes vãos em edificações, além de apresentar melhor estabilidade dimensional (STARK et al., 2010)

O LVL é utilizado principalmente como flanges de vigas em forma de I (I-joist) com camada interna de compensado ou OSB (Oriented Strand Board), e como vergas de portas e janelas na construção de habitações. Em pranchões utilizados em andaimes, utilizações não-estruturais, como na fabricação de esquadrias, escadas e na fabricação de móveis (APA, 2016). Bem como, na construção de pontes de madeira, em que permanece com excelente trabalhabilidade com o emprego de preservativos (RITTER, 1990).

Internacionalmente espécies como Douglas fir, red oak, yellow poplar e poplar têm sido utilizadas na produção de LVL (VLOSKY, et al., 1994). No entanto, para a geração de produtos mais competitivos visando o mercado brasileiro, podem-se empregar novas fontes de matéria-prima, como a espécie Hovenia dulcis Thunb. (uva do japão) e Corymbia citriodora Hook. Pois, ainda não se tem estudos a respeito dessas espécies na produção de lâminas e posterior manufatura de painéis LVL.

A uva do japão é nativa da China, Japão e Coréias, podendo ser encontrada além de sua região de ocorrência no sudeste da Ásia, norte da Argentina, Paraguai e no Brasil, de forma espontânea ou presente em plantios agrícolas. De usos múltiplos, sua madeira é de média densidade $(0,50$ a 0,72 $\left.\mathrm{g} / \mathrm{m}^{3}\right)$, de grã direita, apresenta boa trabalhabilidade (CARVALHO, 1994). Também com uma ampla variedade de utilidades, a espécie Corymbia é cultivada por pequenos e médios produtores rurais em todo o Brasil. De ocorrência natural no leste da Austrália, sua grã é direita podendo ser eventualmente ondulada, a madeira possui densidade entre $0,95 \mathrm{~g} / \mathrm{cm}^{3}$ a $1,01 \mathrm{~g} / \mathrm{cm}^{3}$, além de apresentar boa trabalhabilidade sendo utilizada para diversas finalidades de uso (REIS, et al., 2013).

Em 2012, fabricantes de LVL da América do Norte produziram mais de 1,2 milhões de metros cúbicos em 18 diferentes fábricas (NORTH AMERICAN LAMINATED VENEER LUMBER, 2013). Historicamente, o LVL possui margens de lucro elevadas e, como resultado de melhorias na produção e subsequente redução de custos, essas margens devem permanecer atrativas para esse mercado (VLOSKY, et al., 1994).

Dessa forma, esse trabalho tem como objetivo avaliar a influência do posicionamento das lâminas de Corymbia citriodora e Hovenia dulcis em flexão estática flatwise e edgewise e cisalhamento na linha de cola antes e após fervura em painéis estruturais LVL.

\section{Material e Métodos}

\section{Obtenção e preparo da matéria-prima}

As árvores utilizadas na laminação foram provenientes de povoamentos homogêneos de Hovenia dulcis e Corymbia citriodora pertencentes a Fundação Estadual de Pesquisa Agropecuária (FEPAGRO Florestas), localizada em Santa Maria, RS. As lâminas foram obtidas de três árvores de cada espécie com DAP (diâmetro altura do peito - 1,3 m) entre 28 $\mathrm{cm}$ e $35 \mathrm{~cm}$. Foi utilizado somente o primeiro torete de cada árvore com comprimento de $1,15 \mathrm{~m}$. Os toretes foram processados em torno desenrolador no mesmo dia e local da coleta das árvores. Em seguida, as lâminas foram transportadas ainda verdes em forma de bobinas, as quais 
foram seccionadas para obtenção de lâminas individuais de dimensões de 380 x 480 x 1,0 mm; resultando em 70 lâminas de cada espécie.

Foi realizada uma pré-secagem das lâminas em estufa laboratorial de circulação de ar a $40^{\circ} \mathrm{C}$ para a retirada excessiva da umidade. Na sequência, foram transferidas para a câmara climatizada do LPF $\left(20^{\circ} \mathrm{C}\right.$ e $65 \%$ de umidade relativa) até alcançarem umidade de equilíbrio higroscópico com o ambiente $(\sim 12 \%)$. A determinação do teor de umidade (TUe) e massa específica (MEe) das lâminas seguiu as recomendações das normas da ASTM D 2395 e ASTM D 4442, respectivamente.

\section{Delineamento experimental}

Foram avaliados seis tratamentos com três repetições cada, perfazendo um total de dezoito painéis LVL manufaturados. Cada composição foi formada por cinco lâminas de cada espécie e com mistura das mesmas, formando os tratamentos puros e mistos, respectivamente. Ao final, as dimensões idealizadas para os painéis LVL foram de $38 \mathrm{~cm} \mathrm{x}$ $48 \mathrm{~cm}$ x $5 \mathrm{~mm}$ (largura, comprimento e espessura, aproximadamente).

A identificação de cada painel LVL com sua respectiva composição está apresentada na Figura 1. Os conjuntos foram representados por CCCCC, $\mathrm{CHCHC}, \mathrm{HCCCH}, \mathrm{CHHHC}$, $\mathrm{HCHCH}, \mathrm{HHHHH}$ para quesito de comparação.
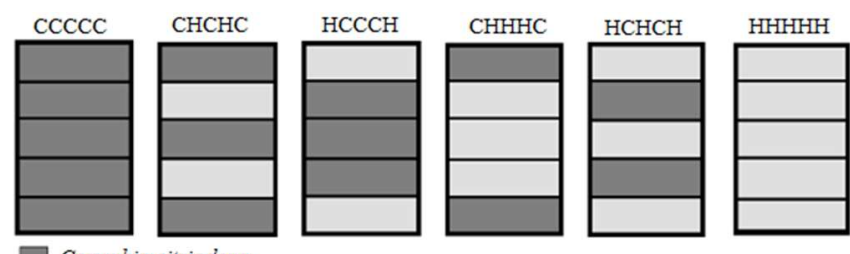

Corymbia citriodora

Hovenia dulcis

Figura 1. Delineamento experimental dos tratamentos LVL. Figure 1. Experimental design of LVL treatments.

\section{Critérios de classificação das lâminas e}

\section{montagem dos painéis}

Primeiramente as lâminas foram classificadas visualmente em lâminas A (ausência de defeitos), lâminas B (presença de nós) e lâminas $\mathrm{C}$ (presença de defeitos como nós, rachaduras e/ ou fendas). Após isso, para a montagem dos painéis LVL todas as lâminas foram posicionadas no mesmo sentido da grã umas das outras. Para as lâminas que formaram as capas e contracapas dos painéis LVL, seguiu-se uma distribuição em ordem crescente de massa específica a partir da média das lâminas cuja classificação visual era A. Porém, quando não haviam lâminas de classificação A, eram utilizadas as lâminas das classificações seguintes. Assim como para as camadas intermediárias, no entanto, em ordem decrescente de distribuição. Já para o miolo das composições, quando houvesse eram utilizadas as lâminas remanescentes das classificações visuais anteriores, como também as lâminas classificadas visualmente por $\mathrm{C}$, em ordem crescente de massa específica a partir da média. Todas as lâminas foram posicionadas paralelamente umas às outras.

Para a colagem das lâminas foi utilizado o adesivo fenolformaldeído, conhecido comercialmente como Cascophen HL - 2090 (52\% de teor de sólidos). Além disso, utilizou-se a farinha de casca de coco micropulverizada como material de enchimento e como extensor a farinha de trigo, além da água. A proporção para a formação da mistura empregada como adesivo consistiu de 100 partes da resina para 5 partes dos demais constituintes. O teor de umidade das lâminas foi estabelecido entre 4 e 6\%, e a gramatura utilizada foi de 200 $\mathrm{g} / \mathrm{m}^{2}$ aplicada em linha simples.

O tempo de aplicação do adesivo nas lâminas correspondentes ao primeiro painel foi de aproximadamente 5 minutos. Após isso, os painéis foram prensados a frio por um período de 1 hora em prensa manual com uma pressão 
apenas para que houvesse contato entre as lâminas e o adesivo Por fim, os painéis foram prensados a $145^{\circ} \mathrm{C}$ em prensa hidráulica da marca Omeco, com pressão específica de 14 $\mathrm{kgf} / \mathrm{cm}^{2}$, por um período de tempo estimado de 6 minutos para a cura do adesivo (dois minutos por milímetro + dois minutos de tempo base).

\section{Ensaios físicos e mecânicos}

Após a confecção dos painéis LVL, os mesmos foram marcados e esquadrejados nas dimensões recomendadas conforme a norma do respectivo ensaio. Assim, para os ensaios de teor de umidade e massa específica aparente, seguiram-se as recomendações das normas ASTM D $4442 \mathrm{e}$ ASTM D 2395, respectivamente.

Para o ensaio de flexão estática na posição flatwise, utilizou-se a norma ASTM D3043. Assim, o vão entre apoios foi de 48 vezes a espessura das amostras, ficando estabelecido em $19 \mathrm{~cm}$ e a velocidade de ensaio calculada de acordo com a norma, em $2 \mathrm{~mm} /$ minuto. Já para a posição edgewise, a norma foi adaptada devido a espessura final dos painéis LVL. Assim, as dimensões ficaram em $47 \mathrm{~cm}$ (comprimento) $\mathrm{x} 4 \mathrm{~mm}$ (base) x $1 \mathrm{~cm}$ (altura), vão de apoio em $48 \mathrm{~cm}$ e velocidade de $5,8 \mathrm{~mm} / \mathrm{min}$. No total foram testadas 2 amostras por painel LVL, totalizando 6 amostras por tratamento.

Para o ensaio de resistência ao cisalhamento na linha de cola foi utilizada a norma ASTM D 905-08. Em que para a realização do ensaio de cisalhamento os corpos de prova foram adaptados devido a espessura fina do painel, o que é permitido segundo a norma. As linhas de cola testadas foram entre Hovenia dulcis x Hovenia dulcis, Corymbia citriodora $\mathrm{x}$ Corymbia citriodora e Hovenia dulcis x Corymbia citriodora. Dessa forma, foram confeccionados 12 corpos de prova para cada tratamento, em que foram ensaiados sob velocidade de aplicação de carga de $0,6 \mathrm{~mm} /$ minuto. A resistência ao cisalhamento $\left(\mathrm{em} \mathrm{kgf} / \mathrm{cm}^{2}\right)$ foi calculada através da divisão da carga máxima suportada (carga de ruptura, em kgf) pela área da lâmina de madeira colada submetida ao cisalhamento (em $\left.\mathrm{cm}^{2}\right)$.

Além disso, da mesma maneira que o ensaio a seco (em temperatura ambiente) citado anteriormente foram ensaiados 6 corpos de prova para cada combinação, no entanto os mesmos foram fervidos por um período de 4 horas. Após isso, foram secos em estufa de circulação de ar a $63^{\circ} \mathrm{C}$ para diminuir o teor de umidade em $8 \%$. Em seguida foram novamente fervidos por mais 4 horas, transcorrido esse tempo foram resfriados em água fria $\left(21^{\circ} \mathrm{C}\right)$ e testados úmidos.

A análise estatística dos dados foi realizada através do delineamento inteiramente casualizado (DIC). Após seguidos os pressupostos estatísticos de homogeneidade das variâncias (Bartlett) e normalidade (Shapiro-Wilk) os dados foram interpretados por meio da análise de variância (ANOVA) em 5\% de significância. Em caso de rejeição da hipótese nula foi realizado o teste LSD (least significant difference) de Fisher em nível de 5\% de significância.

\section{Resultados e Discussão}

Para as lâminas de Corymbia citriodora e Hovenia dulcis foram obtidos teores de umidade de $11,4 \%$ e $10,7 \%$ e massa específica aparente de $898 \mathrm{~kg} / \mathrm{m}^{3}$ e $566 \mathrm{~kg} / \mathrm{m}^{3}$, respectivamente. Já conforme a norma brasileira NBR 7190 (1997) a madeira de Corymbia citriodora apresenta o valor médio da massa específica aparente de $999 \mathrm{~kg} / \mathrm{m}^{3}$. Segundo Carvalho (1994), em estudos com a madeira de Hovenia dulcis a $15 \%$ de umidade encontrou os valores de massa específica aparente entre 500 e $720 \mathrm{~kg} / \mathrm{m}^{3}$. O que demonstra que a densidade das lâminas deste estudo apresenta-se próxima a esses valores de densidade.

Os painéis LVL apresentaram um valor médio de TUe de $10,5 \%$, base seca. Os valores médios de massa específica estão 
apresentados na Tabela 1, assim como os resultados de flexão estática na posição flatwise.

Tabela 1. Resultados da massa específica aparente (MEe) e teste de flexão estática na posição flatwise: módulo de elasticidade (MOE) e módulo de ruptura (MOR).

Table 1. Results of density and static bending strength on flatwise position: modulus of elasticity (MOE) and modulus of rupture (MOR).

\begin{tabular}{cccc}
\hline \multirow{2}{*}{ Tratamento } & \multirow{2}{*}{ MEe $\left(\mathrm{kg} / \mathrm{m}^{3}\right)$} & \multicolumn{2}{c}{ Posição flatwise } \\
& & MOE $(\mathrm{MPa})$ & MOR $(\mathrm{MPa})$ \\
\hline LVL1 & $1022_{\mathbf{A}}{ }^{4,08}$ & $16946_{\mathbf{A}^{14,24}}$ & $144_{\mathbf{A}^{7,08}}$ \\
LVL2 & $922_{\mathbf{B}}{ }^{2,56}$ & $13551_{\mathbf{B}^{3,76}}$ & $131_{\mathbf{A B}}{ }^{2,91}$ \\
LVL3 & $950_{\mathbf{B}}{ }^{2,40}$ & $13572_{\mathbf{B}}{ }^{15,22}$ & $134_{\mathbf{A B}^{5,86}}$ \\
LVL4 & $932_{\mathbf{B}}{ }^{2,21}$ & $17409_{\mathbf{A}^{9,04}}$ & $139_{\mathbf{A B}^{7,74}}$ \\
LVL5 & $953_{\mathbf{B}}{ }^{6,18}$ & $15720_{\mathbf{A B}^{15,08}}$ & $137_{\mathbf{A B}}{ }^{13,68}$ \\
LVL6 & $818_{\mathbf{C}^{3,22}}$ & $9964_{\mathbf{C}^{4,47}}$ & $121_{\mathrm{C}^{7,55}}$ \\
\hline
\end{tabular}

Letras maiúsculas iguais não diferem estatisticamente ao nível de 5\% de significância; os valores acima e à direita das letras refere-se ao coeficiente de variação $(\mathrm{CV})$. Em que: $\mathrm{C}$ representa as lâminas de Corymbia citriodora e $\mathrm{H}$ as lâminas de Hovenia dulcis.

Devido a baixa espessura das lâminas presentes nos painéis LVL $(\sim 1,0 \mathrm{~mm})$ houve um aumento da massa específica final dos mesmos em relação à massa específica da madeira das espécies que os deram origem. Isso ocorreu porque o fenol-formaldeído possui maior densidade que as lâminas de madeira. Somado a isso, painéis produzidos com lâminas mais finas apresentaram maior proporção de adesivo em relação à madeira no volume final do que aqueles com lâminas mais grossas. Ainda, há uma redução da espessura das lâminas depois da densificação dos painéis, mais influente naquelas com menor massa específica e na posição externa dos painéis devido à pressão dos pratos da prensa. Esses resultados estão em conformidade com Melo; Menezzi (2014) que verificou um aumento da massa específica com a redução da espessura das lâminas em LVLs de Schizolobium amazonicum.

Dependendo da espécie, as faixas de calor e pressão utilizadas nas indústrias, há uma diminuição de espessura de
$5 \%$ a $10 \%$, aproximadamente, para teores de umidade de até 10\%. Dessa forma, a densidade de um painel multilaminado é usualmente maior que a madeira sólida (KOLLMANN et al., 1975).

Houve um aumento de 13,9\% e 16,5\% quando foram colocadas duas lâminas de Corymbia citriodora nas posições intermediárias e externas, $\mathrm{HCHCH}$ e $\mathrm{CHHHC}$, respectivamente em relação ao tratamento $\mathrm{HHHHH}$. Já quando foram colocadas duas lâminas de Hovenia dulcis houve uma diminuição de 10,8\% e 7,6\% para os tratamentos $\mathrm{CHCHC}$ e $\mathrm{HCCCH}$, respectivamente, em relação ao tratamento CCCCC. Assim, percebe-se que a colocação de duas ou três lâminas de maior densidade proporcionou uma maior amplitude de acréscimo na densidade do painel do que a amplitude de decréscimo quando colocadas duas ou três lâminas de menor densidade. Somado a isso, nota-se que quando as lâminas foram intercaladas entre as duas espécies não importando a posição em que ocupam no painel os resultados de MEe foram semelhantes. É possível que a baixa espessura dessas lâminas justifique a não diferenciação entre as camadas, aliado a isso a mistura entre as espécies no mesmo painel proporcionou um balanceamento entre as diferentes composições.

Os tratamentos puros CCCCC (cinco lâminas de Corymbia citriodora) e HHHHH (cinco lâminas de Hovenia dulcis) apresentaram resultados significativamente distintos tanto em MOE quanto em MOR em flexão estática na posição flatwise. O tratamento CCCCC, apesar de significativa diferença de densidade do tratamento CHHHC (capas e contracapas com lâminas de Corymbia citriodora) em MOE obtiveram resultados semelhantes entre si, juntamente com o tratamento $\mathrm{HCHCH}$, o que pode estar associado possivelmente à baixa qualidade de colagem entre as lâminas de Corymbia citriodora (vide resultados dos ensaios de resistência ao cisalhamento). 
Ainda, a colocação de lâminas de maior massa específica nas capas e contracapas dos tratamentos LVL não evidenciou diferenças nos resultados de MOE. Visto que, os tratamentos $\mathrm{CHCHC}$ e CHHHC apresentaram resultados distintos, o que pode ser explicado pela pequena distância entre as lâminas das capas e contracapas em relação às lâminas das camadas intermediárias ( 1 mm). Além disso, destaca-se os resultados superiores do tratamento $\mathrm{CHHHC}$ em relação ao tratamento CCCCC. Fatores como maior massa específica das lâminas que compõe as capas e contracapas dos painéis $\mathrm{CHHHC}$ influenciaram nesses resultados.

Também, verificou-se nos resultados de MOE, que as lâminas de Corymbia citriodora posicionadas nas camadas de miolo não foram influentes, pois o tratamento $\mathrm{HCCCH}$ é equivalente estatisticamente ao tratamento $\mathrm{HCHCH}$. Além disso, os tratamentos $\mathrm{CHHHC}$ e $\mathrm{CHCHC}$ apresentaram resultados semelhantes, demonstrando que não há influência do posicionamento de lâminas de Corymbia citriodora nas camadas de miolo. Conforme Müller (2015), isso pode ser devido a essas lâminas estarem localizadas em região de tensão mínima em relação à linha neutra, dessa forma, são menos exigidas mecanicamente.

Para as camadas intermediárias, percebe-se que as mesmas também não influenciaram nos resultados de MOE. Uma vez que, os tratamentos $\mathrm{CHCHC}$ e $\mathrm{HCCCH}$ não se diferenciaram entre si, bem como os tratamentos $\mathrm{CHHHC} \mathrm{e}$ $\mathrm{HCHCH}$.

Os valores médios de MOR dos tratamentos LVL mistos foram semelhantes entre si e corroboram com os resultados de suas respectivas MEes. Demonstrando que houve maior influência da MEe dos painéis LVL nos resultados de resistência à flexão do que as diferenças de MEe entre as posições das lâminas. Assim, parece que as lâminas de maior MEe não proporcionaram maior resistência dependendo da posição em que ocupam na composição do painel.
Iwakiri et al. (2010), estudando as propriedades de painéis LVL observou que a colocação de lâminas de Eucalyptus saligna (maior massa específica) em relação às lâminas de Schizolobium amazonicum e Pinus taeda nas camadas externas de painéis LVL evidenciaram um aumento nos valores médios de MOE e MOR, tanto na posição edgewise quanto na flatwise. Müller (2015) também verificou semelhantes comportamentos em painéis LVL de Eucalyptus saligna e Pinus taeda. Assim, percebe-se que a baixa espessura das lâminas obtidas neste estudo dificultou a avalição da uma influência da massa específica das lâminas conforme as posições em que ocupam no painel.

Além disso, verifica-se que apenas nos tratamentos LVL puros refletiram-se os padrões das diferenças estatísticas de MOR em relação ao MOE. Apesar dos ganhos em rigidez dos tratamentos LVL CHHHC a resistência dos mesmos permaneceu semelhante entre os demais tratamentos mistos.

$\mathrm{Na}$ Tabela 2 podem-se verificar os resultados dos ensaios de flexão estática na posição edgewise para os seis tratamentos LVL, assim como a massa específica correspondente a cada um deles.

Assim como mencionado por Müller (2015), o que poderia influenciar na posição edgewise seria a massa específica de cada tratamento, uma vez que a carga é aplicada igualmente a todas as lâminas do conjunto. Assim, percebe-se que os tratamentos $\mathrm{CHCHC}$ e $\mathrm{HCHCH}$ refletiram os resultados de $\mathrm{MOE}$ em relação à $\mathrm{MEe}$, bem como os tratamentos puros CCCCC e HHHHH. Para os tratamentos $\mathrm{HCCCH}$ e $\mathrm{CHHHC}$ nota-se que os resultados de MOE foram distintos apesar de possuírem semelhantes MEes. Possivelmente o baixo desempenho do tratamento CHHHC deve-se a colocação de mais de duas lâminas de Hovenia dulcis, as quais possuem menor MEe em relação as lâminas de Corymbia citriodora. 
Tabela 2. Resultados do teste de flexão estática na posição edgewise: módulo de elasticidade (MOE) e módulo de ruptura (MOR).

Table 2. Results of static bending strength on edgewise position: modulus of elasticity (MOE) and modulus of rupture (MOR).

\begin{tabular}{cccc}
\hline \multirow{2}{*}{ Tratamento } & \multirow{2}{*}{ MEe $\left(\mathrm{kg} / \mathrm{m}^{3}\right)$} & \multicolumn{2}{c}{ Posição edgewise } \\
& & MOE $(\mathrm{MPa})$ & MOR $(\mathrm{MPa})$ \\
\hline LVL1 & $1022_{\mathrm{A}}^{4,08}$ & $14928_{\mathrm{A}}{ }^{10,27}$ & $137_{\mathrm{B}}{ }^{7,40}$ \\
LVL2 & $922_{\mathrm{B}}^{2,56}$ & $12611_{\mathrm{B}}^{9,55}$ & $149_{\mathrm{AB}}{ }^{9,88}$ \\
LVL3 & $950_{\mathrm{B}}{ }^{2,40}$ & $14469_{\mathrm{A}}{ }^{10,76}$ & $160_{\mathrm{A}}{ }^{4,73}$ \\
LVL4 & $932_{\mathrm{B}}^{2,21}$ & $9953_{\mathrm{C}_{11,86}}$ & $123_{\mathrm{C}^{7,45}}$ \\
LVL5 & $953_{\mathrm{B}}{ }^{6,18}$ & $12115_{\mathrm{B}}^{9,35}$ & $121_{\mathrm{C}^{8,69}}$ \\
LVL6 & $818 \mathrm{C}^{3,22}$ & $9214_{\mathrm{C}}^{13,63}$ & $122_{\mathrm{C}}^{6,31}$ \\
\hline
\end{tabular}

Letras maiúsculas iguais não diferem estatisticamente ao nível de 5\% de significância; os valores acima e à direita das letras refere-se ao coeficiente de variação $(\mathrm{CV})$. Em que: C representa as lâminas de Corymbia citriodora e $\mathrm{H}$ as lâminas de Hovenia dulcis.

De maneira geral, percebe-se que houve influência das lâminas que compõe os tratamentos e não somente da $\mathrm{MEe}$ dos mesmos. Nota-se ainda que apesar de o tratamento CHCHC possuir uma lâmina a mais de Corymbia citriodora que o tratamento $\mathrm{HCHCH}$ ambos apresentaram semelhantes respostas nos resultados de MOE.

Além disso, percebe-se que houve influência das camadas de miolo, tanto nos resultados de MOE quanto nos de MOR. Uma vez que, o tratamento $\mathrm{CHCHC}$ obteve desempenho superior ao tratamento $\mathrm{CHHHC}$, assim como o tratamento $\mathrm{HCCCH}$ em relação ao tratamento $\mathrm{HCHCH}$.

O comportamento semelhante de $\mathrm{MOE}$ em relação ao MOR apenas é verificado nos tratamentos $\mathrm{HCCCH}, \mathrm{CHHHC}$ e HHHHH. Ainda, os tratamentos com três lâminas de Corymbia citriodora ( $\mathrm{CHCHC}$ e $\mathrm{HCCCH}$ ) foram os que obtiveram melhor desempenho em MOR em relação aos demais, assim como o tratamento CCCCC. No entanto, na posição edgewise o desempenho do tratamento CCCCC foi inferior ao tratamento $\mathrm{HCCCH}$, o que não foi observado nos resultados de MOE. Possivelmente irregularidades na espessura das lâminas que compõe o tratamento LVL, além de defeitos como fendas, podem ter comprometido o desempenho do tratamento CCCCC em MOR, visto que em MOE os resultados foram semelhantes; o mesmo ocorreu para o tratamento $\mathrm{HCHCH}$.

Os valores médios de MOR foram maiores na posição edgewise em relação à flatwise para os tratamentos LVL com três lâminas de Corymbia citriodora ( $\mathrm{CHCHC}$ e $\mathrm{HCCCH})$. O contrário para os tratamentos LVL com duas lâminas de Corymbia citriodora (CHHHC e HCHCH).

Lara Palma; Ballarin (2011) estudando painéis LVL compostos de 25 lâminas de 2,4 mm de espessura cada de Eucalyptus grandis encontraram valores médios para MOE e MOR de 13114 MPa e 75,06 MPa, respectivamente, em flexão estática (flatwise). Sendo a MEe desses painéis foi de 690 $\mathrm{kg} / \mathrm{m}^{3}$, os quais foram colados com resina fenol-formaldeído com gramatura de $350 \mathrm{~g} / \mathrm{m}^{2}$ por linha dupla de colagem. Já para a posição edgewise encontraram valores médios de MOE de $15871 \mathrm{MPa}$ e de MOR de 88,63 MPa. Nota-se que apesar de maior proporção de adesivo e madeira no volume final desses painéis em relação aos deste estudo, como no caso do tratamento CCCCC o valor médio de MOE foi superior na posição flatwise, no entanto, para a posição edgewise o valor foi $6,3 \%$ menor. O que pode ter influência das características que as lâminas que compõe o tratamento CCCCC possuem e não somente da massa específica do painel. No caso do MOR em ambas as posições foram superiores, provavelmente devido a influência da massa específica da espécie Corymbia citriodora.

Müller (2015) pesquisou sobre painéis LVL formados por cinco lâminas de $3 \mathrm{~mm}$ cada de Eucalyptus saligna e Pinus taeda colados com fenol-formaldeído (gramatura de $200 \mathrm{~g} / \mathrm{m}^{2}$ ). Assim, encontrou para os painéis LVL formados por apenas lâminas de Eucalyptus saligna massa específica aparente de $898 \mathrm{~kg} / \mathrm{m}^{3}$, para a posição flatwise MOE de 22385 MPa e MOR de $168 \mathrm{MPa}$. Já para a posição edgewise o valor 
médio de MOE $20014 \mathrm{MPa}$ e MOR $132 \mathrm{MPa}$. Isso se deve a maior proporção de madeira no volume final do que dos painéis do tratamento CCCCC, apesar de MEe de ambos os estudos serem semelhantes entre si.

A associação norte-americana APA/EWS PRL-501 (2000) estabelece valores como limites inferiores de rigidez e resistência para cinco tipos de classes de exigência. Em MOE, acima de $14479 \mathrm{MPa}$ caracteriza a classe mais alta, sendo ela a primeira classe, tanto para a posição flatwise quanto edgewise. Para MOR na posição flatwise é de 44,89 $\mathrm{MPa}$, também para a primeira classe, já para edgewise é de 52,36 MPa. Dessa forma, no caso de MOR todos os tratamentos LVL se enquadram na classe mais alta da APA/EWS, tanto na posição flatwise quanto na edgewise. Na posição flatwise, em MOE os tratamentos CCCCC e CHHHC se enquadram na $1^{\text {a }}$ classe, sendo os tratamentos $\mathrm{HCHCH}$ na $2^{\text {a }}$ classe, $\mathrm{CHCHC} \mathrm{e}$ HCCCH na $3^{\text {a }}$ classe. No entanto, HHHHH não se enquadra em nenhuma das classes da APA/EWS, nas duas posições. Para a posição edgewise, o tratamento CCCCC se enquadra na $1^{\text {a }}$ classe, $\mathrm{HCCCH}$ na $2^{\text {a }}$ classe, $\mathrm{HCHCH}$ na $3^{\text {a }}$ classe, $\mathrm{CHCHC}$ na $4^{\text {a }}$ classe e CHHHC em nenhuma das classes de rigidez da referida norma. Cabe salientar que os valores dessas classes se referem à altura dos painéis LVL menor que $8,89 \mathrm{~cm}(3,5$ ”) e teores de umidade menor que $16 \%$, assim como mencionado por Müller (2015).

Green et al. (1999) determina os coeficientes de variação para a madeira em diversas propriedades, no caso do módulo de elasticidade e módulo de ruptura $22 \%$ e $16 \%$, respectivamente, todos os tratamentos LVL estão abaixo dos valores limites apresentados. Demonstrando bom desempenho e homogeneidade entre as amostras dentro de cada tratamento.

\section{Ensaio de cisalhamento na linha de cola}

Os ensaios de cisalhamento na linha de cola antes e após fervura foram realizados para três tipos de colagem: Corymbia citriodora x Corymbia citriodora $(\mathrm{C} \times \mathrm{C})$, Corymbia citriodora $\mathrm{x}$ Hovenia dulcis $(\mathrm{C} \times \mathrm{H})$ e Hovenia dulcis $\mathrm{x}$ Hovenia dulcis $(\mathrm{H}$ x H) (Tabela 3).

Tabela 3. Resistência ao cisalhamento na linha de cola a seco e após fervura.

Table 3. Shear strength in the glue line test dry and after boiling.

\begin{tabular}{ccc}
\hline \multirow{2}{*}{ Composições } & $\begin{array}{c}\text { Cisalhamento a } \\
\text { seco }\end{array}$ & $\begin{array}{c}\text { Cisalhamento após } \\
\text { fervura }\end{array}$ \\
\cline { 2 - 3 } & \multicolumn{2}{c}{ Tensão de ruptura $(\mathrm{MPa})$} \\
\hline $\mathrm{C} \times \mathrm{C}$ & $5,7_{\mathrm{B}}{ }^{11,7}$ & $2,9_{\mathrm{B}}{ }^{10,5}$ \\
$\mathrm{C}$ x H & $5,8_{\mathrm{B}}{ }^{8,5}$ & $2,9_{\mathrm{B}}{ }^{6,61}$ \\
$\mathrm{H}$ x H & $10,2_{\mathrm{A}}{ }^{7,2}$ & $3,8_{\mathrm{A}}{ }^{3,4}$ \\
\hline
\end{tabular}

Em cada linha estão apresentados os valores médios de cada tratamento, sendo que médias com letras maiúsculas iguais não diferem estatisticamente ao nível de $5 \%$ de significância de erro; o valor acima e à direita das letras é o coeficiente de variação $(\mathrm{CV})$.

Observa-se que as composições $\mathrm{C}$ x C e C x H apresentaram resultados semelhantes, tanto nos ensaios a seco quanto após fervura das amostras. Dentre os arranjos testados o melhor desempenho, para os dois tipos de ensaios, foi encontrado na composição formada pelas lâminas de Hovenia dulcis $(\mathrm{H} \mathrm{x} \mathrm{H)}$.

Buligon et al. (2015) encontrou valores médios de 7,16 MPa e 3,04 MPa para resistência ao cisalhamento em ensaios a seco e após fervura, respectivamente, de composições formadas por lâminas de Pinus elliottii coladas com fenolformaldeído na gramatura de $190 \mathrm{~g} / \mathrm{m}^{2}$ em linha simples. Percebe-se excelente performance das composições formadas pelas lâminas de Hovenia dulcis, visto que a massa específica dessas lâminas comparadas com a do trabalho citado foi semelhante.

A NBR 7190 (1997) apresenta o valor médio de 10,7 MPa de resistência ao cisalhamento paralelo à grã da madeira de 
Corymbia citriodora. O valor médio encontrado para essa propriedade está abaixo em 53\% do apresentado pela norma. Ozarska (1999) comenta que nas espécies australianas de eucalipto de alta massa específica, a presença de extrativos dificultou a eficiência da colagem usando fenol-formaldeído.

Além disso, não necessariamente deve haver uniformidade na espessura entre as lâminas que compõe os painéis. No entanto, variações muito grandes de espessura ao longo da lâmina podem causar falhas de adesão, prejudicando o desempenho do painel (CLARK, 1965). Segundo o mesmo autor, superfícies irregulares, gordura, sujeira ou poeira podem seriamente enfraquecer a eficiência da adesão entre lâminas.

Principalmente para madeiras densas e com o objetivo de se obter lâminas grossas o processo de aquecimento das toras à altas temperaturas antes da laminação, produz lâminas mais firmes e de espessura mais homogênea. Cabe ressaltar, que não foi realizado o processo de aquecimento das toras antes da laminação, o que também pode ter contribuído para esses resultados. Além disso, pode haver a presença de defeitos nas lâminas como: rugosidade, fendas de laminação, lâminas felpudas, fibras escamadas (no lado fechado da lâmina), ondulações, espessura desuniforme, no sentido perpendicular às fibras e lâminas mais grossas nas pontas do que no centro. Os quais podem ser causados devido à pressão insuficiente da contra-faca, a tora estar muito fria ou muito quente, faca sem fio, ângulo da barra de compressão grande, pressão da barra de compressão muito alta, ângulo da faca muito grande ou pequeno e, até mesmo, distorção por aquecimento da faca e barra de compressão (SUCHSLAND; JANKOWSKY, 1978); (LUTZ, 1974).

Neste estudo foi verificado a presença de defeitos importantes como alguns (fendas, rugosidades e espessuras das bordas diferentes do que no centro da lâmina) dos mencionados anteriormente nas lâminas de Corymbia citriodora. O que possivelmente tenham influenciado na eficiência da colagem das lâminas dessa espécie. Justificando o baixo valor de resistência ao cisalhamento em relação aos encontrados na literatura. Isso pode ter ocorrido devido a principalmente a insuficiente afiação da faca e distorção da barra de compressão durante a laminação.

Palma; Ballarin (2011) encontraram para painéis LVL de Eucalyptus grandis (lâminas de 2,4 mm de espessura com massa específica aparente de $649 \mathrm{~kg} / \mathrm{m}^{3}$ ) utilizando o adesivo fenol-formaldeído valor médio de 5,96 MPa para resistência ao cisalhamento paralelo a linha de cola. Valor esse $18 \%$ abaixo do encontrado pela norma NBR 7190 para a madeira dessa espécie. Uma vez que, a massa específica das lâminas utilizadas para esse ensaio foi de $649 \mathrm{~kg} / \mathrm{m}^{3}$, assim, possivelmente houve influência de fatores intrínsecos da espécie e das características externas das lâminas nesses resultados, bem como para os resultados deste estudo.

Apesar disso, a APA/EWS PRL - 501 (2001) apresenta valores de resistência ao cisalhamento para painéis estruturais LVL, com o limite inferior da classe mais alta apresenta o valor de 900 psi (6,2 MPa). Assim, apenas a composição U x U se encaixaria na classe mais alta, no entanto, as demais composições se enquadram na última classe dessa norma, que apresenta um valor de 695 psi (4,8 MPa).

\section{Conclusões}

Verificou-se fraca influência do posicionamento de lâminas de maior MEe nas camadas externas, intermediárias e de miolo na posição flatwise, o que não é observado na posição edgewise.

Apesar da baixa espessura das lâminas de ambas as espécies em estudo $(\sim 1 \mathrm{~mm})$ a resistência em ambas as posições dos painéis LVL apresentaram excelentes resultados. Somado a isso, o tratamento com apenas lâminas de Corymbia citriodora obteve o melhor desempenho mecânico em ambas 
as posições e se enquadra na classe mais alta da norma APA/EWS PRL - 501. No entanto, ocorreu o oposto para o tratamento com apenas lâminas de Hovenia dulcis. Os tratamentos mistos, com exceção do tratamento CHHHC em MOE na posição edgewise, se enquadram em uma das classes da respectiva norma.

A composição formada por lâminas de Hovenia dulcis apresentou desempenho superior em resistência ao cisalhamento na linha de cola em relação a composição de lâminas de Corymbia citriodora. Porém, ambas apresentam potencial para serem utilizadas na produção de painéis LVL estruturais.

\section{Referências}

AMERICAN SOCIETY FOR TESTING AND MATERIALS ASTM. D2395, Standard Test Methods for Density and Specific Gravity (Relative Density) of Wood and Wood-Based Materials, ASTM International, West Conshohocken, PA, 2014.

AMERICAN SOCIETY FOR TESTING AND MATERIALS ASTM. D3043-00, Standard Test Methods for Structural Panels in Flexure, ASTM International, West Conshohocken, PA, 2011.

\section{AMERICAN SOCIETY FOR TESTING AND MATERIALS -} ASTM. D4442, Standard Test Methods for Direct Moisture Content Measurement of Wood and Wood-Based Materials, ASTM International, West Conshohocken, PA, 2016.

AMERICAN SOCIETY FOR TESTING AND MATERIALS ASTM. D905-08, Standard Test Method for Strength Properties of Adhesive Bonds in Shear by Compression Loading, ASTM International, West Conshohocken, PA, 2013.

APA/EWS-ENGINEERED WOOD ASSOCIATION/ENGINEERED WOOD SYSTEM. PRL 501: Performance Standard for APA EWS Laminated Veneer Lumber. Engineered Wood System, Form EWS B310.Washington, 2000. 8p.

APA-ENGINEERED WOOD ASSOCIATION. Engineered Wood - Construction guide. n. E30W, p.7, 2016. Disponível em: <https://apawood.org/>. Acesso em: 03 set 2016.
ASSOCIAÇÃO BRASILEIRA DE NORMAS TÉCNICAS. NBR 7190: Projeto de estruturas de madeira. Rio de Janeiro, 1997, 107 p.

BULIGON, E. A.; HASELEIN, C.R.; GATTO, D.A.; SANTINI, E.J.; RAUBER, R.; BERGER, C. Propriedades físicomecânicas de painéis de lâminas paralelas reforçadas com PRFV. Ciência Florestal, Santa Maria, v. 25, n. 3, p.729-739, jul.-set., 2015.

CARVALHO, P. E. R. Ecologia, silvicultura e usos da uva-dojapão (Hovenia dulcis, Thunberg). Circular Técnica EMBRAPA, n. 23, p.24, 1994.

CLARK, W. Veneering and Wood Bending in the Furniture industry. Pergamon Press Ltd. 1 ed., p. 120, 1965.

GREEN, D. W.; WINANDY, J. E.; KRETSCHMANN, D. E. Mechanical properties of Wood. In: Wood Handbook - Wood as an engineering material. Gen. Tech. Rep. FPL-GTR-113. Madison, WI: U.S. Department of Agriculture, Forest Service, Forest Products Laboratory, 1999. cap 4, p. 4-23.

IWAKIRI, S.; MATOS, J. L. M. de; PINTO, J. A.; VIANA, L. C.; SOUZA, M. M. de; TRIANOSKI, R.; ALMEIDA, V. C. Produção de painéis laminados unidirecionais-LVL com lâminas de Schizolobium amazonicum, Eucalyptus saligna e Pinus taeda. Cerne, Lavras, v. 16, n. 4, p. 557-563, 2010.

KOLLMANN, F. P. K.; CÔTÉ Jr., W. A. Principles of science and technology - I. Solid wood. New York: Springer-Velag, p. $592,1968$.

LARA PALMA, H. A.; BALLARIN, A. W. Propriedades físicas e mecânicas de painéis LVL de Eucallyptus grandis. Ciência Florestal, v. 21, n. 3, p. 559-566, 2011.

LUTZ, J. F. Techniques for peeling, slicing and drying veneer. Madison, WI: U.S. Department of Agriculture, Forest Service, Forest Products Laboratory. Research Paper FPL 228, p. 64, 1974.

MELO, R. R.; DEL MENEZZI, C. H. S. Influence of veneer thickness on the properties of LVL from Paricá (Schizolobium amazonicum) plantation trees. European Journal of Wood and Wood Products, v. 72, p. 191-198, 2014.

MÜlLER, M. T.; HASELEIN, C. R; DE MELO, R. R.; STANGERLIN, D. M. Influência de diferentes combinações de lâminas de Eucalyptus saligna e Pinus taeda. Ciência Florestal, v. 25, n. 1, p. 153-164, 2015. 
NORTH AMERICAN LAMINATED VENEER LUMBER. Environmental Product Declaration - American Wood Council. p. 3, 2013. Disponível em $<$ http://awc.org/pdf/greenbuilding/epd/AWC-EPD-LVL1307.pdf>. Acesso em: 10 out. 2015.

OZARSKA, B. A review of the utilization of hardwoods for LVL. Wood Science and Technology, v. 33, p. 341-351, 1999.

RITTER, M A. Types of timber bridges. In: Timber bridges, design, construction, inspection, and maintenance. Washington, DC: Department of Agriculture, Forest Service, Forest Products Laboratory, 1990. cap. 2, p. 6.

STARK, N. M.; CAI, Z.; CARLL, C. Wood based composites materials panel products, glued-laminated timber, structural composite lumber, and wood-nonwood, composite materials. In: Wood Handbook - Wood as an engineering material. General Technical Report FPL-GTR-190. Madison, WI: U.S. Department of Agriculture, Forest Service, Forest Products Laboratory, 2010. cap. 11, p. 11-28.

SUCHSLAND, O.; JANCOWSKY, P. I. A produção de lâminas por densenrolamento. Circular Técnica, n. 33. IPEF, 1978. 